Article

\title{
The Rotational Barrier in Ethane: A Molecular Orbital Study
}

\author{
Ramiro F. Quijano-Quiñones ${ }^{1}{ }^{*}$, Mariana Quesadas-Rojas ${ }^{1}$, Gabriel Cuevas ${ }^{2}$ and \\ Gonzalo J. Mena-Rejón ${ }^{1}$
}

1 Laboratory of Pharmaceutical Chemistry, Faculty of Chemistry, Autonomous University of Yucatan, 41 No. 421 Col. Industrial, C.P. 97150, Merida, Yucatan, Mexico;

E-Mails: mariana_quesadas@yahoo.com.mx (M.Q.-R.); mrejon@uady.mx (G.J.M.-R.)

2

Institute of Chemistry, National Autonomous University of Mexico, Circuito Exterior, Ciudad Universitaria, C.P. 04510 Mexico D.F., Mexico; E-Mail: gecgb@unam.mx

* Author to whom correspondence should be addressed; E-Mail: ramiro.quijano@uady.mx; Tel./Fax: +52-999-922-5711.

Received: 27 March 2012; in revised form: 12 April 2012 / Accepted: 12 April 2012 /

Published: 20 April 2012

\begin{abstract}
The energy change on each Occupied Molecular Orbital as a function of rotation about the C-C bond in ethane was studied using the B3LYP, mPWB95 functional and MP2 methods with different basis sets. Also, the effect of the ZPE on rotational barrier was analyzed. We have found that $\sigma$ and $\pi$ energies contribution stabilize a staggered conformation. The $\sigma_{\mathrm{s}}$ molecular orbital stabilizes the staggered conformation while the $\sigma_{\mathrm{s}}^{*}$ stabilizes the eclipsed conformation and destabilize the staggered conformation. The $\pi_{\mathrm{z}}$ and $\pi_{\mathrm{z}}^{*}$ molecular orbitals stabilize both the eclipsed and staggered conformations, which are destabilized by the $\pi_{\mathrm{v}}$ and $\pi_{\mathrm{y}}^{*}$ molecular orbitals. The results show that the method of calculation has the effect of changing the behavior of the energy change in each Occupied Molecular Orbital energy as a function of the angle of rotation about the $\mathrm{C}-\mathrm{C}$ bond in ethane. Finally, we found that if the molecular orbital energy contribution is deleted from the rotational energy, an inversion in conformational preference occurs.
\end{abstract}

Keywords: staggered conformation; ethane; rotational barrier; molecular orbital; DFT 


\section{Introduction}

The existence of a rotational barrier of $2.875 \mathrm{kcal} \cdot \mathrm{mol}^{-1}$ about the $\mathrm{C}-\mathrm{C}$ bond in ethane has been known for many years [1-9]. There are two main effects that have been regarded as responsible for this rotational barrier: A steric repulsion in the eclipsed conformation [2-4,9-14] and an enhanced stabilization of the staggered conformation due to hyperconjugation [5,8,15-22].

The steric effect has its origin in the fact that atoms in molecules occupy a certain amount of space, resulting in changes in shape, energy, and reactivity. It is an essential concept in chemistry, biochemistry, and pharmacology, influencing rates and energies of chemical reactions, impacting structure, dynamics, and function of enzymes, and to a degree, governing how and at what rate a drug molecule interacts with a receptor. Different approaches have been proposed to quantify the steric effect. For example, Shubin Liu recently proposed an energy partition scheme under the framework of Density Functional Theory (DFT) $[23,24]$. In this scheme the total energy density functional is decomposed into three independent contributions from steric, electrostatic, and quantum effects. This scheme was used to explore the internal rotation barrier of various molecules [14,25-27]. In particular, they analyzed the origin of the rotation barrier in ethane and concluded that the eclipse conformer possesses a large steric repulsion than the staggered conformer [14] in support of the steric repulsion as the cause for the preferred staggered conformation of ethane. However, to date there is no consensus about which is the origin of the steric effect and about the method to use to quantify it. For example, Weisskopf [28] attributed it to the "kinetic energy pressure" in atoms and molecules, whereas others [9,10,12,13,29] employed the quantum contribution from the Pauli Exclusion Principle [30-34]. Therefore, due to the different approximations used for the calculation of steric effect it is not possible to draw final conclusions from these calculations. Nevertheless, the steric repulsion still remains the most popular explanation for the preferred staggered conformation of the ethane [2-4,9-14].

Alternatively, the possible role of hyperconjugation effect in the ethane rotation barrier has been conjectured for many years [21,22]. Hyperconjugation corresponds to the interaction between an occupied bond orbital and a vicinal unoccupied antibond orbital, which results in an occupied delocalized orbital and in stabilization of the system. Lately, Pophristic and Goodman [15] have argued that the rotational energy barrier cannot be explained by steric repulsion between vicinal $\mathrm{C}-\mathrm{H}$ bonds in the eclipsed conformation. They proposed that the staggered conformation results from hyperconjugation effects. In this conformation, the $\mathrm{C}-\mathrm{H}$ bonds have a favorable disposition for the interaction of the $\sigma^{*}$ antibonding orbital of one $\mathrm{C}-\mathrm{H}$ unit with the corresponding occupied $\sigma$ bonding orbital at the other side. They have used a natural bond orbital (NBO) [35], analysis to prove the existence of hyperconjugation effects by deleting the $\sigma-\sigma^{*}$ interactions. The conclusion of this method is supported by other similar studies [16-20]. Nevertheless, the NBO analysis for the ethane conformations has been challenged by different authors because NBO does not leave the electron density and the energy unchanged, thus causing an energy lowering that affects the final result $[10,36]$.

Therefore, there is no definite explanation for the driving force of the preferred ethane conformation mainly due to the different approximations used for the calculation of steric and hyperconjugative effects, in addition to the difficulty of their simultaneous calculation and because hyperconjugation, 
steric repulsion, and possibly some other effects coexist entangled in the ethane molecule. In consequence, different authors obtain different amounts of steric and hyperconjugation effects.

In order to contribute to the understanding of the conformational driving force in ethane, we propose an alternative point of view based on a systematic analysis of its Molecular Orbitals (MOs), the most basic concept in conformation, to assign the different MOs to each of the preferred conformations and estimate the overall net effect by subtracting the molecular orbital energy from the total energy during the rotation about the $\mathrm{C}-\mathrm{C}$ bond in the ethane. In addition, we propose to study the effect that this theoretical model has on the behavior of energy of the MOs as a function of angle of rotation about $\mathrm{C}-\mathrm{C}$ bond in ethane.

Based on these considerations, we carried out the analysis of electronic and structural properties of ethane as a function of the $\mathrm{C}-\mathrm{C}$ angle $(\varphi)$ rotation. The DFT (B3LYP, mPWB95) and MP2 methods with $6-31 G(d, p), 6-31+G(d, p)$, and $6-31++G(d, p)$ basis sets were used to evaluate the effects of these models in the ethane rotational molecular orbital energy and the relationship to the ethane-preferred conformation. The B3LYP functional was used because of its wide application to calculate electronic structure, reaction and activation energies. However, there is evidence that the B3LYP method usually underestimates barrier heights [37,38]. Additionally, the third generation mPWB95 functional was applied since recent studies in small systems have shown that it yields more reliable results than the B3LYP functional $[39,40]$. We compared the results calculated with DFT and those calculated with MP2 theory since it its known that B3LYP and MP2 give errors in opposite direction in the energy for organic molecules [41]. In addition, we compared the basis 6-31G(d,p), 6-31+G(d,p), and $6-31++\mathrm{G}(\mathrm{d}, \mathrm{p})$ in order to evaluate the effect of the addition of polarization and diffusion to the basis set. Finally, in order to evaluate the effect of zero-point energy (ZPE) on rotational barrier, the calculations were performed with- or without consideration of ZPE. All energies reported with zero-point corrections are not scaled for comparative purpose.

The aim of this work is to contribute to understanding of the contribution of each of the molecular orbitals in ethane to the rotational barrier and its overall net effect. In addition, we propose analyze the effect that theoretical model has on the behavior of energy of the MOs as a function of angle of rotation about $\mathrm{C}-\mathrm{C}$ bond in ethane. To the best of our knowledge, this is the first study of energy changes on each orbital in ethane by different methods.

\section{Results and Discussion}

The Kohn-Sham total energy rotation $\left(E_{\text {rot }}\right)$ for conversion of ethane from staggered to eclipsed conformation was calculated in the gas phase and the geometries were fully optimized. In the applied models the total energy of ethane is calculated as a function of the torsion angle $\varphi$, obtaining an energy minimum at the staggered $\left(E_{s}\right)$ conformation and a maximum at the eclipsed $\left(E_{e}\right)$ conformation. The energy difference between $E_{e}$ and $E_{s}\left(E_{e}-E_{s}\right)$ is the calculated rotational barrier $\left(\Delta E_{\text {rot }}\right)$.

Regarding evaluation of the of ZPE effect on the rotational barrier it is important to notice that if ZPE is not included (Table 1), the MP2 model seems to overestimate the rotational barrier while the DFT model underestimates it. In addition, the inclusion of diffuse functions provokes a decrease of the rotational barrier. Finally, the B3LYP/6-31G(d,p) level of theory allowed to obtain the closest rotational barrier $\left(2.8020 \mathrm{kcal} \cdot \mathrm{mol}^{-1}\right)$ to the experimental value $\left(2.875 \mathrm{kcal} \cdot \mathrm{mol}^{-1}\right)$. Similar results are 
observed when ZPE was included for MP2/6-31G(d, p) level of theory, which estimates a rotational barrier of $2.9116 \mathrm{kcal} \cdot \mathrm{mol}^{-1}$.

Table 1. Calculated values of $\Delta \mathrm{E}_{\mathrm{rot}}\left(\mathrm{kcal} \cdot \mathrm{mol}^{-1}\right)$.

\begin{tabular}{cccccccccc}
\hline & B3G & B3+G & B3++G & MPG & MP+G & MP++G & MP2G & MP2+G & MP2++G \\
\hline$\Delta \mathrm{E}_{\text {rot }}$ & 2.803 & 2.732 & 2.736 & 2.752 & 2.673 & 2.683 & 3.025 & 2.966 & 2.981 \\
$\Delta \mathrm{E}_{\text {rot }}^{\text {PE }}$ & 2.541 & 2.466 & 2.472 & 2.504 & 2.422 & 2.435 & 2.912 & 2.799 & 2.761 \\
\hline
\end{tabular}

Labels B3, MP correspond to the B3LYP and mPWB95 functionals, MP2 correspond to the Moller-Plesset perturbation theory of order 2 and the symbols $\mathrm{G},+\mathrm{G}$, and $++\mathrm{G}$ represent the basis set 6-31G(d, p), 6-31+G(d, p), and 6-31++G(d, p), respectively.

The lengths of the $\mathrm{C}-\mathrm{C}$ and $\mathrm{C}-\mathrm{H}$ bonds were obtained as a function of $\varphi$. It is interesting to note that when the conformation goes from eclipsed $\left(\varphi=0^{\circ}\right)$ to staggered $\left(\varphi=60^{\circ}\right)$ the $\mathrm{C}-\mathrm{C}$ bond length decreases slightly, while the $\mathrm{C}-\mathrm{H}$ bonds increase slightly. The percent decrease of $\mathrm{C}-\mathrm{C}$ bond length varies from 0.9 when the calculation is performed with B3LYP/6-31G $(\mathrm{d}, \mathrm{p})$ to 0.85 if the MP2/6-31G(d,p) method is applied. For the C-H bond length increase the percent variation is from 0.089 [MP2/6-31G(d,p)) to $0.1(\mathrm{~B} 3 \mathrm{LYP} / 6-31 \mathrm{G}(\mathrm{d}, \mathrm{p})]$. Thus, rotation about the $\mathrm{C}-\mathrm{C}$ bond generates a small effect over the geometry of ethane.

Ethane consists of two carbon and six hydrogen atoms sharing nine filled MOs. The valence molecular orbital configuration appropriate to the $\mathrm{D}_{3 \mathrm{~d}}$ symmetry staggered conformation is $\left(2 a_{1 g}\right)^{2}\left(2 a_{2 u}\right)^{2}\left(e_{g}\right)^{4}\left(3 a_{2 u}\right)^{2}\left(e_{u}\right)^{4}$ while that of the $\mathrm{D}_{3 \mathrm{~h}}$ symmetry of the eclipsed conformer is $\left(2 a_{1}^{\prime}\right)^{2}\left(2 a^{\prime \prime}\right)^{2}\left(e^{\prime \prime}\right)^{4}\left(3 a^{\prime \prime}{ }_{2}\right)^{2}\left(e^{\prime}\right)^{4}$. The contributions from " $a$ " category orbitals represent the $s$ orbital interactions and those from " $e$ " category orbitals represent $p$ orbital interactions [15,42]. For this reason the MOs are called $\sigma_{\mathrm{s}}, \sigma_{\mathrm{s}}^{*}, \pi_{\mathrm{v}}, \pi_{\mathrm{z}}, \sigma_{\mathrm{x}}, \pi_{\mathrm{y}}^{*}$, and $\pi_{\mathrm{z}}^{*}$ respectively. Figure 1 also shows the core orbital's and the $\pi$ character of the degenerate $\pi_{\mathrm{v}}, \pi_{\mathrm{z}}, \pi_{\mathrm{y}}^{*}$, and $\pi_{\mathrm{z}}^{*}$ molecular orbitals, and the $\sigma$ character of the $\sigma_{\mathrm{s}}, \sigma_{\mathrm{s}}^{*}$ and $\sigma_{\mathrm{x}}$ molecular orbitals at B3LYP/6-31+G(d,p) level of theory.

Figure 1. Filled molecular orbitals of ethane calculated with B3LYP/6-31+G(d, p) theoretical model.

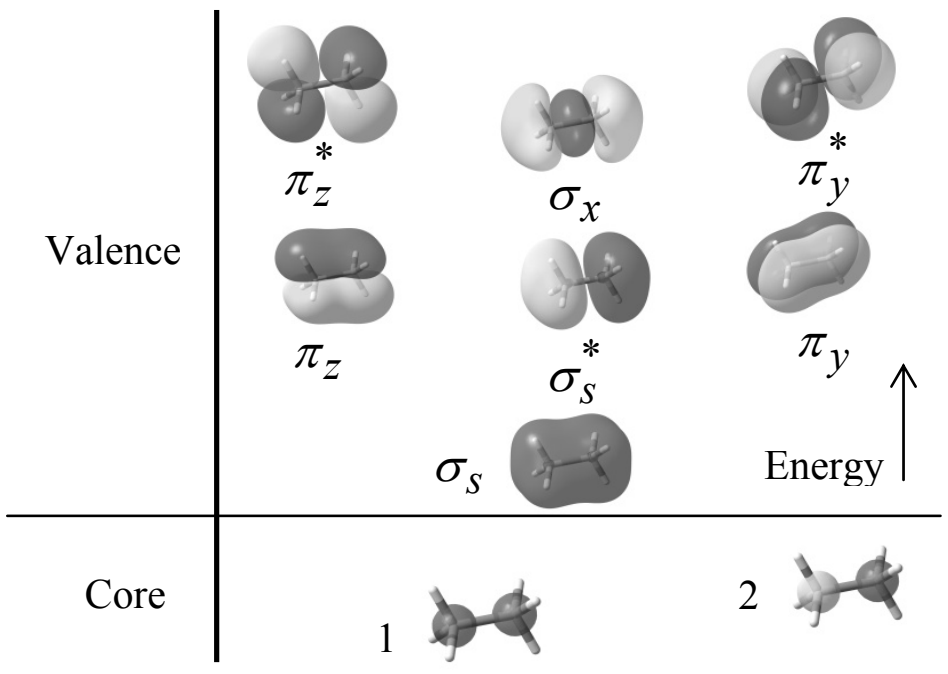


Along the potential energy surface (PES) the MOs are changing, but, for simplicity we retain the labels of the MOs in its evolution as a function of angle of rotation. The energy changes in the MOs, calculated as a function of $\varphi$ are shown in Figures 2, 3 and 4. Since there are no MOs associated to the MP2 energies, we plotted the energy of the canonical Hartree-Fock (HF) MOs associated to each MP2. Each MO core showed a minimum energy at the staggered conformation (Figure 2). The hydrogen atoms have MOs with large $s$ character. The $\sigma_{\mathrm{s}}$ and $\sigma_{\mathrm{s}}^{*}$ orbitals present mainly $\mathrm{C}(1 s)$ and $\mathrm{C}(2 p)$ character and are main contributors to the $\mathrm{C}-\mathrm{C}$ bond strength. All the $\sigma_{\mathrm{s}}$ bonding orbitals exhibited a minimum energy at staggered conformation (Figure 2). It is possible to observe that the energy changes between eclipsed and staggered conformations fluctuate from $2.667 \mathrm{kcal} \cdot \mathrm{mol}^{-1}(\mathrm{HF} / 6-31 \mathrm{G}(\mathrm{d}, \mathrm{p}))$ down to $1.626 \mathrm{kcal} \cdot \mathrm{mol}^{-1}$ (mPWB95/6-31+G(d,p)).

Figure 2. Energy of the core, $\sigma_{\mathrm{s}}$ and $\sigma_{\mathrm{s}}^{*}$ occupy MOs of ethane as a function of the rotational angle, calculated with the entire theoretical model studied in this work. Labels B3, MP correspond to the B3LYP and mPWB95 functionals, MP2 correspond to HF theory and the symbols $\mathrm{G},+\mathrm{G}$, and $++\mathrm{G}$ represent the basis set $6-31 \mathrm{G}(\mathrm{d}, \mathrm{p}), 6-31+\mathrm{G}(\mathrm{d}, \mathrm{p})$, and $6-31++G(d, p)$, respectively.
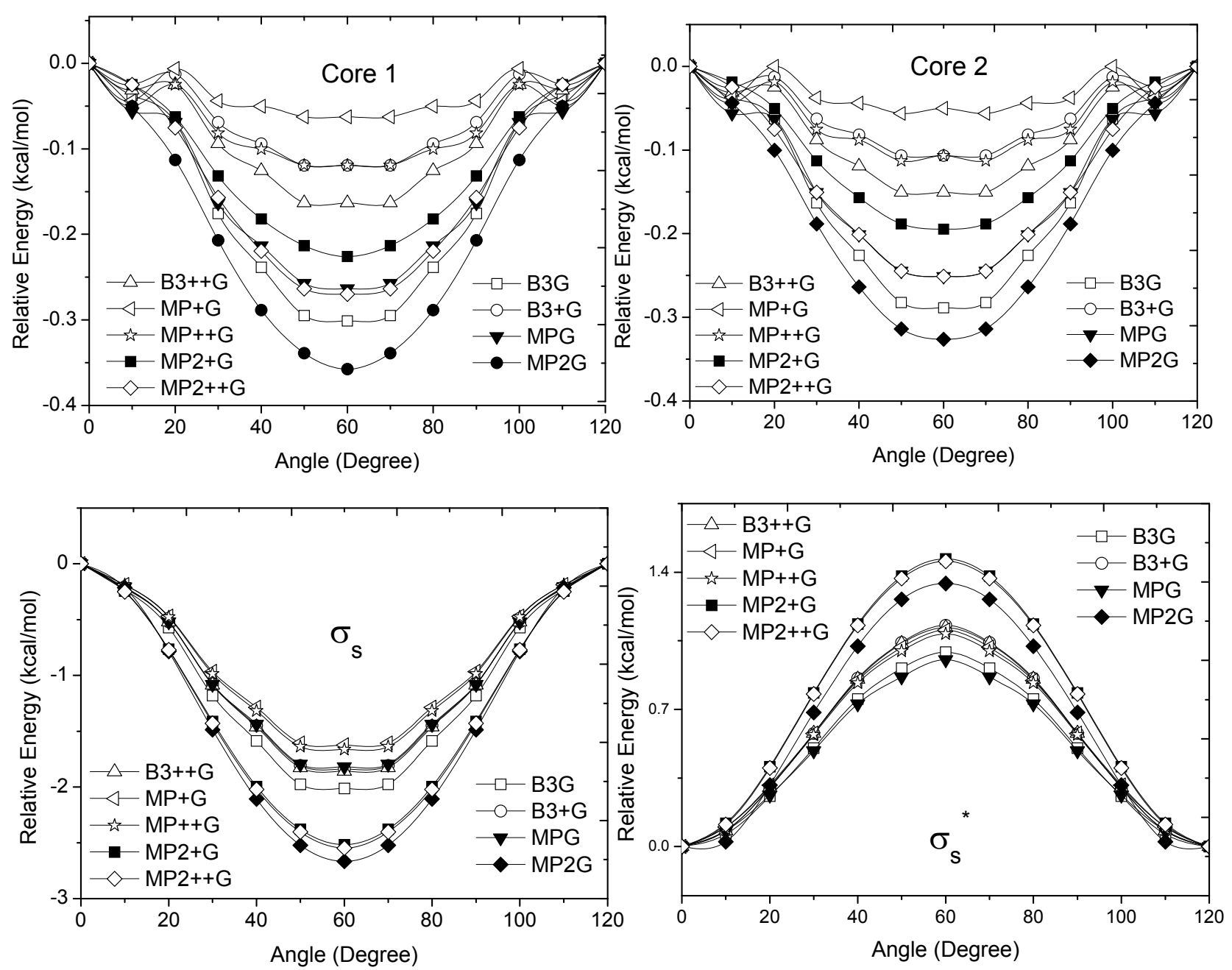

These results show the importance of the $\sigma_{\mathrm{s}}$ orbital bonding for the rotational barrier. Interestingly, the $\sigma_{\mathrm{s}}^{*}$ antibonding molecular orbital presented a minimum energy at the eclipsed conformation and a 
maximum at the staggered conformation with energies that change from $-1.4683 \mathrm{kcal} \cdot \mathrm{mol}^{-1}$ $[\mathrm{HF} / 6-31 \mathrm{G}(\mathrm{d}, \mathrm{p})]$ down to $-0.9538 \mathrm{kcal} \cdot \mathrm{mol}^{-1}[\mathrm{mPWB} 95 / 6-31+\mathrm{G}(\mathrm{d}, \mathrm{p})]$. The negative sign of the value reveals that the $\sigma_{\mathrm{s}}^{*}$ molecular orbital had a minimum energy at $\varphi=0^{\circ}$, which stabilized the eclipsed conformer.

The bonding $\pi_{\mathrm{v}}$ and $\pi_{\mathrm{z}}$, and antibonding $\pi_{\mathrm{y}}^{*}$ and $\pi_{\mathrm{z}}^{*}$ sets have a large $\mathrm{H}(1 s)$ character and $\mathrm{C}(2 p)$ character. These MOs are mainly associated to the vicinal hyperconjugative delocalization interactions between the methyl groups. The energy values, calculated for the change from staggered to eclipsed conformation in the orbitals $\pi_{\mathrm{z}}, \pi_{\mathrm{v}}, \pi_{\mathrm{z}}^{*}$ and $\pi_{\mathrm{y}}^{*}$, vary from 0.7216 [mPWB95/6-31G(d,p)], 0.7467 [mPWB95/6-31+G(d,p)], 1.1044 [mPWB95/6-31+G(d,p)] and $1.1546 \mathrm{kcal}^{-\mathrm{mol}^{-1}}$ [mPWB95/6-31+G(d,p)] to $1.267[\mathrm{HF} / 6-31 \mathrm{G}(\mathrm{d}, \mathrm{p})], 1.794$ [HF/6-31G(d,p)], 1.2989 [B3LYP/6-31G(d,p)] and $1.3114 \mathrm{kcal} \cdot \mathrm{mol}^{-1}$ [B3LYP/6-31+G(d,p)], respectively (Figures 3 and 4).

Figure 3. Energy of the $\pi_{\mathrm{v}}, \pi_{\mathrm{z}}, \sigma_{\mathrm{x}}$ and $\pi_{\mathrm{y}}^{*}$ occupy MOs of ethane as a function of the rotational angle, calculated with the entire theoretical model studied in this work. Labels B3, MP correspond to the B3LYP, and mPWB95 functionals, MP2 correspond to the HF theory and the symbols $\mathrm{G},+\mathrm{G}$ and $++\mathrm{G}$ represent the basis set 6-31G(d, p), 6-31+G(d, p), and $6-31++\mathrm{G}(\mathrm{d}, \mathrm{p})$, respectively.
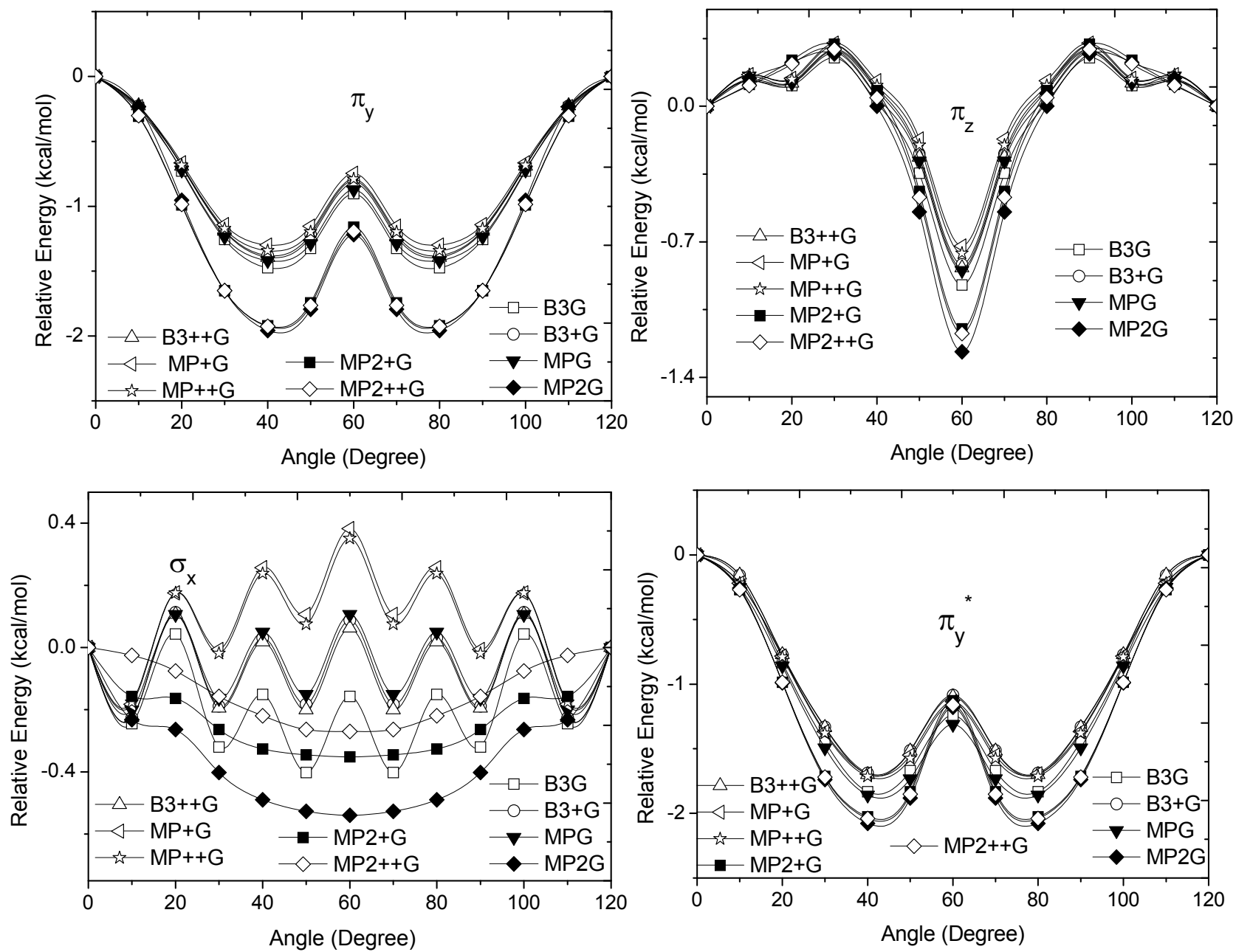
Figure 4. Energy of the $\pi_{\mathrm{z}}^{*}$ occupied MO of ethane as a function of the rotational angle, calculated with the entire theoretical model studied in this work. Labels B3, MP correspond to the B3LYP and mPWB95 functionals, MP2 correspond to the HF theory and the symbols $\mathrm{G},+\mathrm{G}$, and $++\mathrm{G}$ represent the basis set $6-31 \mathrm{G}(\mathrm{d}, \mathrm{p}), 6-31+\mathrm{G}(\mathrm{d}, \mathrm{p})$, and $6-31++\mathrm{G}(\mathrm{d}, \mathrm{p})$, respectively.

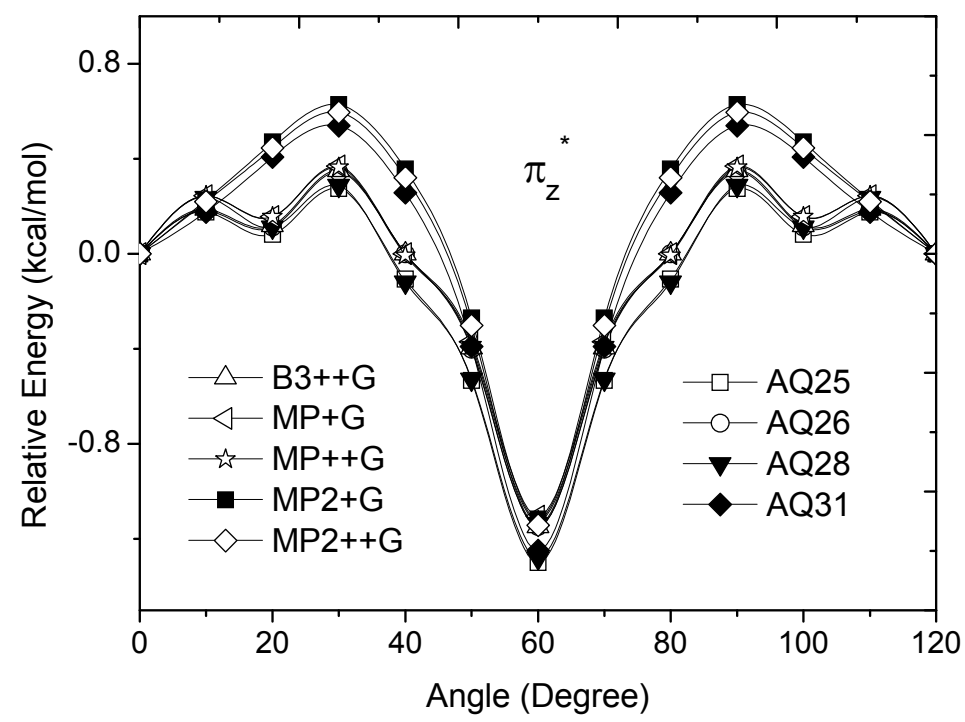

The calculated energy change for the studied MOs was equivalent to $\Delta \mathrm{E}_{\text {rot }}$, demonstrating the role of these orbitals for the rotational barrier of ethane. However, for all models, the bonding and antibonding $\pi_{\mathrm{v}}$ and $\pi_{\mathrm{y}}^{*}$ orbitals showed a smaller local maximum energy at staggered rather than at eclipsed conformation. Additionally, the energy presented a symmetric double minimum structure close to $\varphi=40^{\circ}$ and $\varphi=80^{\circ}$. These results demonstrate that the energy changes in the $\pi_{\mathrm{v}}$ and $\pi_{\mathrm{y}}^{*}$ MOs destabilize the staggered conformer.

The total energy of the $\pi_{\mathrm{z}}$ and $\pi_{\mathrm{z}}^{*}$ MOs showed an asymmetric double minimum structure at $\varphi=0^{\circ}$ (eclipsed conformation; local minimum) and $\varphi=60^{\circ}$ (staggered conformation; global minimum) in addition to two maxima close to $\varphi=20^{\circ}$ and $\varphi=100^{\circ}$. Thus, the energy change in the $\pi_{\mathrm{z}}$ and $\pi_{\mathrm{z}}^{*}$ MOs stabilizes both conformations. It is important to remark, however, that calculations based on DFT provided two energy minima close to $\varphi=40$ and $\varphi=100^{\circ}$, which were not found when the HF model was used.

Finally, the molecular orbital $\sigma_{\mathrm{x}}$, exhibited large $\mathrm{H}(1 s)$ and $\mathrm{C}(2 p)$ characters. The calculated values for the change between eclipsed and staggered conformations, showed variations from $-0.3827 \mathrm{kcal} \cdot \mathrm{mol}^{-1}$ $[\mathrm{HF} / 6-31 \mathrm{G}(\mathrm{d}, \mathrm{p})]$ to $0.5396 \mathrm{kcal} \cdot \mathrm{mol}^{-1}[\mathrm{mPWB} 95 / 6-31 \mathrm{G}(\mathrm{d}, \mathrm{p})]$ (Figure 3). In this case, calculations was carried out using the HF model showed an energy minimum at $\varphi=60^{\circ}$; revealing that this $\mathrm{MO}$ contributes to stabilize the staggered conformation. On the other hand, calculations based on DFT indicated irregular changes in energy, while only the B3LYP/6-31G(d,p) model predicts that the staggered conformation is stabilized by the contribution of the $\sigma_{\mathrm{x}}$ orbital.

From Figures 3 and 4, we can see that the DFT and HF methods predict different behaviors in the molecular orbital energy of the $\sigma_{\mathrm{x}}, \pi_{\mathrm{z}}$, and $\pi_{\mathrm{z}}^{*}$ MOs. The total electronic energy of the molecular orbital $\mathrm{E}_{\text {elec }}^{\mathrm{MO}}$ was calculated as a function of $\varphi$, considering two electrons for each MO. In all cases, the minimum and maximum values of both $\mathrm{E}_{\text {elec }}^{\mathrm{MO}}$ and $\mathrm{E}_{\text {rot }}$ coincided. The overall net effect of the $\mathrm{E}_{\text {elec }}^{\mathrm{MO}}$ in 
conformational preference of the ethane can be estimated if the difference in energy between the $\mathrm{E}_{\text {elec }}^{\mathrm{MO}}$ in the eclipsed conformation and $\mathrm{E}_{\text {elec }}^{\mathrm{MO}}$ in staggered conformation $\left(\Delta \mathrm{E}_{\text {elec }}^{\mathrm{MO}}\right)$ is compared to $\Delta \mathrm{E}_{\text {rot }}$. We can see from the Table 2 that the $\Delta \mathrm{E}_{\text {elec }}^{\mathrm{MO}}$ was higher than the $\Delta \mathrm{E}_{\text {rot }}$ for all methods and basis set, in spite of the difference found between the different models in the behavior of the MO as a function of $\varphi$. This indicates that the energy difference between $\mathrm{E}_{\text {rot }}$ and $\mathrm{E}_{\text {elec }}^{\mathrm{MO}}$ as a function of $\varphi$ produced an inversion of the minimum. Accordingly if $\mathrm{E}_{\text {elec }}^{\mathrm{MO}}$ is subtracted, the preference of $\mathrm{E}_{\mathrm{rot}}$ for the staggered conformer is lost, and the eclipsed conformer becomes more stable. Additionally, we can note that the calculations using the HF model predict a higher $\Delta \mathrm{E}_{\text {elec }}^{\mathrm{MO}}$ than those performed with DFT, while the use of the functional mPWB95 of DFT allowed predict a lower value of $\Delta \mathrm{E}_{\text {elec }}^{\mathrm{MO}}$ (Table 2). Finally, the inclusion of diffuse functions in all calculations resulted in a decrease of $\Delta \mathrm{E}_{\text {elec }}^{\mathrm{MO}}$.

Table 2. Calculated values of $\Delta \mathrm{E}_{\text {elec }}^{\mathrm{MO}}, \sigma\left(\Delta \mathrm{E}_{\text {elec( }(\sigma)}^{\mathrm{MO}}\right)$ and $\pi\left(\Delta \mathrm{E}_{\text {elec }(\pi)}^{\mathrm{MO}}\right)$ contributions compared to $\Delta \mathrm{E}_{\mathrm{rot}}$ and $\Delta \mathrm{E}_{\mathrm{rot}}^{\mathrm{ZPE}}(\mathrm{Kcal} / \mathrm{mol})$.

\begin{tabular}{cccccccccc}
\hline & B3G & B3+G & B3++G & MPG & MP+G & MP++G & MP2G & MP2+G & MP2++G \\
\hline$\Delta \mathrm{E}_{\text {rot }}$ & 2.803 & 2.732 & 2.736 & 2.752 & 2.673 & 2.683 & 3.025 & 2.966 & 2.981 \\
$\Delta \mathrm{E}_{\text {rot }}^{\mathrm{ZPE}}$ & 2.541 & 2.466 & 2.472 & 2.504 & 2.422 & 2.435 & 2.912 & 2.799 & 2.761 \\
$\Delta \mathrm{E}_{\text {elec }}^{\mathrm{MO}}$ & 12.27 & 9.400 & 9.79 & 11.170 & 7.919 & 8.559 & 14.92 & 12.75 & 13.29 \\
$\Delta \mathrm{E}_{\text {elec }(\sigma)}^{\mathrm{MO}}$ & 3.539 & 1.694 & 1.983 & 2.548 & 0.502 & 0.904 & 5.095 & 3.640 & 3.953 \\
$\Delta \mathrm{E}_{\text {elec }(\pi)}^{\mathrm{MO}}$ & 8.735 & 7.706 & 7.806 & 8.622 & 7.417 & 7.656 & 9.827 & 9.111 & 9.337 \\
\hline
\end{tabular}

Labels B3, MP correspond to the B3LYP and mPWB95 functionals, MP2 correspond to the HF theory and the symbols $\mathrm{G},+\mathrm{G}$, and $++\mathrm{G}$ represent the basis set $6-31 \mathrm{G}(\mathrm{d}, \mathrm{p}), 6-31+\mathrm{G}(\mathrm{d}, \mathrm{p})$, and $6-31++G(d, p)$, respectively.

The value of total $\sigma\left(\Delta \mathrm{E}_{\operatorname{elec}(\sigma)}^{\mathrm{MO}}\right)$ and $\pi\left(\Delta \mathrm{E}_{\operatorname{elec}(\pi)}^{\mathrm{MO}}\right)$ contributions to $\Delta \mathrm{E}_{\text {elec }}^{\mathrm{MO}}$ for all used models is shown in Table 2. It was found that when the $\sigma$ contribution is deleted, the conformational preference is reversed. A similar behavior was observed for the total $\pi$ energy contribution to $\Delta \mathrm{E}_{\text {elec }}^{\mathrm{MO}}$. It is important to note that the $\pi$ energy contribution is higher than the $\sigma$ contribution.

\section{Experimental}

The quantum chemical calculation was performed using the GAUSSIAN 09 code [43] The total energy dependence in the torsional angle was calculated using Density Functional Theory (DFT) [44], with a B3LYP, mPWB95 functional and MP2 method [45], and 6-31G(d, p), 6-31+G(d, p) and $6-31++\mathrm{G}(\mathrm{d}, \mathrm{p})$ basis set. The rotation about the central carbon-carbon single bond from eclipsed to staggered conformation was considered at $10^{\circ}$ intervals. Full optimization of $\mathrm{C}-\mathrm{C}$ bond and $\mathrm{CH}_{3}$ geometries of the ethane were carried out in all calculations.

\section{Conclusions}

The calculated rotational energy barriers at different levels show that it is not necessary to incorporate diffusion functions for an accurate description of the energetic barrier in ethane. It is important to note that the B3LYP/6-31G(d,p) model underestimates the value of the rotational barrier 
in ethane, while the MP2/6-31G(d,p) model overestimates it. In addition, the functional mPWB95 predict the worst values for rotational barrier and MP2/6-31+G(d, p) predicts the higher energy changes. We have found that the $\pi_{\mathrm{v}}$ and $\pi_{\mathrm{y}}^{*}$ orbitals showed a smaller local maximum energy at staggered than at eclipsed conformation. In addition, the energy change of the $\pi_{\mathrm{z}}$ and $\pi_{\mathrm{z}}^{*}$ MO's stabilizes the eclipsed and the staggered conformations. The DFT methods predict two energy minima close to $\varphi=40$ and $\varphi=100^{\circ}$. The $\pi_{z}$ and $\pi_{z}^{*}$ orbitals stabilize both conformations. For the $\sigma_{\mathrm{x}} \mathrm{MO}$, the DFT energy changes contribute to stabilize the staggered conformation and shows irregular behavior. In addition, we found that for all models if $\mathrm{E}_{\text {elec }}^{\mathrm{MO}}$ is subtracted from the total energy of the ethane, the conformational preference in ethane is the eclipsed conformer.

\section{Acknowledgments}

The financial support was provided by Facultad de Química of Universidad Autónoma de Yucatán, México (PIFI-FOMES 2007). The authors wish to thank to Leovigildo Quijano for the critical review and the help for document translation, and to Manuel Flores-Arce for English revision of the manuscript.

\section{References and Notes}

1. Kemp, J.D.; Pitzer, K.S. Hindered rotation of the methyl groups in ethane. J. Chem. Phys. 1936, 4, 749.

2. Volhard, K.P.C.; Schore, N.E. Organic Chemistry Structure and Function, 5th ed.; W.H. Freeman and Company: New York, NY, USA, 2007; pp. 79-83.

3. Solomons, T.W.G. Organic Chemistry, 5th ed.; John Wiley \& Sons, Inc.: New York, NY, USA, 1992; p. 59.

4. March, J.; Smith, M.B. March's Advanced Organic Chemistry Reactions, Mechanisms and Structure, 6th ed.; John Wiley \& Sons, Inc.: New Jersey, NJ, USA, 2007; pp. 197-198.

5. Brunck, T.K.; Weinhold, F. Quantum-mechanical studies on the origin of barriers to internal rotation about single bonds. J. Am. Chem. Soc. 1979, 101, 1700-1709.

6. Pitzer, R.M. The barrier to internal rotation in ethane. Acc. Chem. Res. 1983, 16, 207-210.

7. Bader, R.F.W.; Cheeseman, J.R.; Laidig, K.E.; Wiberg, K.B.; Breneman, C. Origin of rotation and inversion barriers. J. Am. Chem. Soc. 1990, 112, 6530-6536.

8. Reed, A.E.; Weinhold, F. Natural bond orbital analysis of internal rotation barriers and related phenomena. Isr. J. Chem. 1991, 31, 277-285.

9. Mo, Y.; Wu, W.; Song, L.; Lin, M.; Zhang, Q.; Gao, J. The magnitude of hyperconjugation in ethane: A perspective from ab initio valence bond theory. Angew. Chem. Int. Ed. Engl. 2004, 43, 1986-1990.

10. Bickelhaupt, F.M.; Baerends, E.J. The case for steric repulsion causing the staggered conformation of ethane. Angew. Chem. Int. Ed. Engl. 2003, 42, 4183-4188.

11. Asturiol, D.; Salvador, P.; Mayer, I. Dissecting the hindered rotation of ethane. ChemPhysChem 2009, 10, 1987-1992.

12 Mo, Y.R.; Gao, J.L. Heoretical analysis of the rotational barrier of ethane. Acc. Chem. Res. 2007, 40, 113-119.

13. Baerends, E.J. The rotation barrier in ethane. Nachr. Chem. 2004, 52, 581. 
14. Liu, S.; Govind, N. Toward understanding the nature of internal rotation barriers with a new energy partition scheme: Ethane and n-butane. J. Phys. Chem. A 2008, 112, 6690-6699.

15. Pophristic, V.; Goodman, L. Hyperconjugation not steric repulsion leads to the staggered structure of ethane. Nature 2001, 411, 565-568.

16. England, W.; Gordon, M.S. Localized charge distributions. I. General theory, energy partitioning, and the internal rotation barrier in ethane. J. Am. Chem. Soc. 1971, 93, 4649-4657.

17. Lowe, J.P. A butane analogue, 3-hexyne, is eclipsed. Science 1973, 179, 527-532.

18. Goodman, L.; Gu, H. Flexing analysis of steric exchange repulsion accompanying ethane internal rotation. J. Chem. Phys. 1998, 109, 72-78.

19. Weinhold, M.F. A new twist on molecular shape. Nature 2001, 411, 539-541.

20. Goodman, L.; Gu, H.; Pophristic, V. Flexing analysis of ethane internal rotation energetic. J. Chem. Phys. 1999, 110, 4268-4275.

21. Mulliken, R.S. Intensities of electronic transitions in molecular spectra. IV. Cyclic dienes and hyperconjugation. J. Chem. Phys. 1939, 7, 339-352.

22. Epiotis, N.D.; Cherry, W.R.; Shaik, S.; Yates, R.L.; Bernardi, E. Topics in Current Chemistry: Structural Theory of Organic Chemistry; Springer-Verlag: Berlin, Germany, 1997.

23. Liu, S. Steric effect: A quantitative description from density functional theory. J. Chem. Phys. 2007, 126, 244103-244107.

24. Liu, S. On the relationship between densities of Shannon entropy and Fisher information for atoms and molecules. J. Chem. Phys. 2007, 126, 191107-191109.

25. Liu, S.; Govind, N.; Pedersen, L.G. Exploring the origin of the internal rotational barrier for molecules with one rotatable dihedral angle. J. Chem. Phys. 2008, 129, 094104-094106.

26. Torrent-Sucarrat, M.; Liu, S.; de Proft, F. Steric effect: Partitioning in atomic and functional group contributions. J. Phys. Chem. A 2009, 113, 3698-3702.

27. Esquivel, R.O.; Liu, S.; Angulo, J.C.; Dehesa, J.S.; Antolín, J.; Molina-Espíritu, M. Fisher information and steric effect: Study of the internal rotation barrier of ethane. J. Phys. Chem. A 2011, 115, 4406-4415.

28. Weisskopf, V.F. Of atoms, mountains, and stars: A study in qualitative physics. Science 1975, 187, 605-612.

29. Weinhold, F. Rebuttal to the Bickelhaupt-Baerends case for steric repulsion causing the staggered conformation of ethane. Angew. Chem. Int. Ed. Engl. 2003, 42, 4188-4194.

30. Luken, W.L.; Beratan, D.N. Localized orbitals and the fermi hole. Theor. Chim. Acta 1982, 61, 265-281.

31. Luken, W.L.; Culberson, J.C. Localized orbitals based on the fermi hole. Theor. Chim. Acta 1984, 66, 279-293.

32. Luken, W.L. Properties of the fermi hole in molecules. Croat. Chem. Acta 1984, 57, 1283.

33. Ludena, E.V.; Ugalde, J.M.; Lopez, X.; Fernandez-Rico, J.; Ramirez, G. A reinterpretation of the nature of the fermi hole. J. Chem. Phys. 2004, 120, 540-547.

34. Cooper, D.L.; Ponec, R.A. One-electron approximation to domain averaged fermi hole analysis. Phys. Chem. Chem. Phys. 2008, 10, 1319-1329.

35. Weinhold, F. Natural Bond Orbital Methods. In The Encyclopedia of Computational Chemistry; Schleyer, P.V.R., Ed.; John Wiley \& Sons: Chichester, UK, 1998; pp. 1792-1811. 
36. Mo, Y. Geometrical optimization for strictly localized structures. J. Chem. Phys. 2003, 119, 1300-1306.

37. Durant, J.L. Evaluation of transition state properties by density functional theory. Chem. Phys. Lett. 1996, 256, 595-602.

38. Lynch, B.J.; Fast, P.L.; Harris, M.; Truhlar, D.G. Adiabatic connection for kinetics. J. Phys. Chem. A 2000, 104, 4811-4815.

39. Zhao, Y.; Pu, J.; Lynch, B.J.; Truhlar, D.G. Tests of second-generation and third-generation density functionals for thermochemical kinetics. Phys. Chem. Chem. Phys. 2004, 6, 673-676.

40. Zhao, Y.; Truhlar, D.G. Design of density functionals that are broadly accurate for thermochemistry, thermochemical kinetics, and nonbonded interactions. J. Phys. Chem. A 2005, 109, 5656-5667.

41. Fărcaşiu, D.; Lukinskas, P.; Pamidighantam, S.V. Bridged and open carbocation structures as a function of the correlation level in ab initio calculations: The 4-methyl-2-pentyl cation. J. Phys. Chem. A 2002, 106, 11672-11675.

42. Gimarc, B.M. Qualitative molecular orbital study of ethane and diborane. J. Am. Chem. Soc. 1973, 95, 1417-1421.

43. Frisch, M.J.; Trucks, G.W.; Schlegel, H.B.; Scuseria, G.E.; Robb, M.A.; Cheeseman, J.R.; Scalmani, G.; Barone, V.; Mennucci, B.; Petersson, G.A.; et al.; Gaussian 09, revision A.1; Gaussian, Inc.: Wallingford, CT, USA, 2009.

44. Kohn W.; Sham, L. Self-consistent equations including exchange and correlation effects. J. Phys. Rev. 1965, 140, A1133-A1138.

45. Lee, C.; Yang, W.; Parr, R.G. Development of the colle-salvetti correlation-energy formula into a functional of the electron density. Phys. Rev. B 1988, 37, 785-789.

Sample Availability: Not available.

(C) 2012 by the authors; licensee MDPI, Basel, Switzerland. This article is an open access article distributed under the terms and conditions of the Creative Commons Attribution license (http://creativecommons.org/licenses/by/3.0/). 